\title{
Instrumental Variables Estimation of Stationary and Nonstationary Cointegrating Regressions
}

\author{
P.M. Robinson* and M. Gerolimetto \\ London School of Economics
}

\begin{abstract}
Discussion paper
No. EM/2006/500

April 2006
\end{abstract}

The Suntory Centre

Suntory and Toyota International Centres for

Economics and Related Disciplines

London School of Economics and Political Science

Houghton Street

London WC2A 2AE

Tel: 02079556679

\footnotetext{
* Research supported by ESRC Grant R000239936. Address correspondence to P.M. Robinson, Department of Economics, London School of Economics, Houghton Street, London WC2A 2AE, United Kingdom; email: p.m.robinson@1se.ac.uk.
} 


\begin{abstract}
Instrumental variables estimation is classically employed to avoid simultaneous equations bias in a stable environment. Here we use it to improve upon ordinary least squares estimation of cointegrating regressions between nonstationary and/or long memory stationary variables where the integration orders of regressor and disturbance sum to less than 1, as happens always for stationary regressors, and sometimes for mean-reverting nonstationary ones. Unlike in the classical situation, instruments can be correlated with disturbances and/or uncorrelated with regressors. The approach can also be used in traditional non-fractional cointegrating relations. Various choices of instrument are proposed. Finite sample performance is examined.
\end{abstract}

Keywords: Cointegration; Instrumental variables estimation; $I(d)$ processes.

JEL Classification: $\mathrm{C} 32$

(C) The author. All rights reserved. Short sections of text, not to exceed two paragraphs, may be quoted without explicit permission provided that full credit, including $\odot$ notice, is given to the source. 


\section{Introduction}

A cointegrating relation of rank $P \geq 1$ between elements of a vector $W_{t}$ of $P+Q$ observables, for $Q \geq 1$, can be written

$$
A W_{t}=U_{t}, \quad t=1,2, \ldots
$$

where $A$ is a $P \times(P+Q)$ matrix of rank $P$ and $U_{t}$ is a $P \times 1$ unobservable sequence. In frequent econometric practice, $U_{t}$ is assumed to be a vector of $I(0)$ variables, and $W_{t}$ a vector of $I(1)$ variables. In that case, under minor additional conditions, Stock (1987) showed that ordinary least squares (OLS) estimates of a reduced form of (1.1) are $n$-consistent, where $n$ is sample size. This outcome does not require orthogonality between the right-hand side variables and $U_{t}$, though the limit distribution is non-standard. Estimates with optimality properties which make fuller use of system information have been devised by Johansen (1991), Phillips (1991), for example; these have mixed normal asymptotics and generate Wald statistics with null $\chi^{2}$ asymptotics. Extensions to allow $W_{t}$ to include deterministic, $I(0)$ or $I(2)$ variables have been developed (see e.g. Johansen, 1991, Phillips and Hansen, 1990).

However, $I(1)$ and $I(2)$ are particular notions of stochastic trending behaviour, while $I(0)$ is a particular notion of stationarity, and the concept of cointegration is in no way tied to them. Cointegration involving $I(d)$ processes, for real-valued $d$, has also been of interest, and has been investigated in a number of empirical studies, starting with Cheung and Lai (1993). To discuss this it is convenient to first give a technical definition of $I(0)$ processes: a vector covariance stationary process $v_{t},-\infty<t<\infty$, is said to be $I(0)$ if it has zero mean and spectral density matrix that is positive definite and continuous at zero frequency. Now define formally

$$
\Delta^{-d}=\sum_{j=0}^{\infty} a_{j}(d) L^{j}, \quad a_{j}(d)=\frac{\Gamma(j+d)}{\Gamma(d) \Gamma(j+1)},
$$

$L$ denoting the lag operator, $\Delta=1-L$ and $\Gamma$ denoting the gamma function, which satisfies $\Gamma(d)=\infty$ for $d=0,-1,-2, \ldots$, and $\Gamma(0) / \Gamma(0)=1$. For any sequence $r_{t},-\infty<t<\infty$, define $r_{t}^{\#}=r_{t} 1(t \geq 1)$, where $1($.$) is the indicator$ function.

We introduce a fairly general concept of an $L \times 1$ vector fractional process $\left(u_{t}^{(1)}, \ldots, u_{t}^{(\ell)}\right)^{\prime}$, where the $u_{t}^{(\ell)}, \ell=1, \ldots, L$, are scalars. If $v_{t}$ is a $K \times 1$ vector $I(0)$ process, $1 \leq K<\infty$, with $k$-th element $v_{k t}$, then for $\ell=1, \ldots, L, \Delta^{-d_{k}^{(\ell)}} v_{k t}^{\#}$ is called a basic fractional, $F\left(d_{k}^{(\ell)}\right)$ process and

$$
u_{t}^{(\ell)}=\sum_{k=1}^{K} \Delta^{-d_{k}^{(\ell)}} v_{k t}^{\#}
$$

is called an $I\left(d^{(\ell)}\right)$ process, for $d^{(\ell)}=\max _{1 \leq k \leq K} d_{k}^{(\ell)} ; d$ is called the "integration order" of an $I(d)$ process. The $F\left(d_{k}^{(\ell)}\right)$ component of (1.3) with the largest $d_{k}^{(\ell)}$ 
dominates with respect to asymptotic theory, so reference to other integration orders is suppressed. The truncation in (1.3) is imposed due to lack of convergence of the series (1.2) when $d \geq \frac{1}{2}$; to be consistent it applies for any $d$, including $d \in\left[0, \frac{1}{2}\right)$, but for such $d$ we refer to an $I(d)$ as being "stationary", while $d=\frac{1}{2}$ is on the boundary between stationarity and nonstationarity, and $d>\frac{1}{2}$ is the "nonstationary" region. We call $\left(u_{t}^{(1)}, \ldots, u_{t}^{(L)}\right)$ an $I\left(d^{(1)}, \ldots, d^{(L)}\right)$ process, for $L<\infty$ (where the "degenerate" case $K<L$ is included).

With this terminology we take $\left(W_{t}^{\prime}, U_{t}^{\prime}\right)$ in $(1.1)$ to be an $I\left(\delta_{1}, \ldots, \delta_{P+Q}, \gamma_{1}, \ldots, \gamma_{P}\right)$ process, where cointegration is expressed by

$$
0 \leq \gamma_{i}<\min _{j: a_{i j} \neq 0} \delta_{j}, \quad i=1, \ldots, P,
$$

where $a_{i j}$ is the $(i, j)$-th element of $A$. Note that (1.1) can result from a linear structural model for $W_{t}$ in terms of unobserved $F(d)$ (or $I(d)$ ) components (cf. Stock and Watson, 1988).

Assuming either parametric or nonparametric autocorrelation in the underlying $I(0)$ sequence, and allowing the $\delta_{i}$ and $\gamma_{i}$ to be unknown, estimates of coefficients of a reduced form of (1.1) with apparently optimal properties have been proposed by Robinson and Hualde (2003), Hualde and Robinson (2004, 2006), in the case $P=Q=1$, and in a rather different setting by Jeganathan (1999) when $P=1, Q>1$ but all $\delta_{i}$ are the same; see also Dolado and Marmol (1997), Kim and Phillips (2000). Extending to general $P, Q$, it seems that when $\delta_{j}>\frac{1}{2}$ for all $j$ (and incorporating knowledge of "overidentifying" zero elements of $A$ ), a non-null $a_{i j}$ can be consistently estimated with rate $n^{\delta_{j}-\gamma_{i}}$ when $\delta_{j}-\gamma_{i}>\frac{1}{2}$, and with rate $n^{\frac{1}{2}}$ when $\delta_{j}-\gamma_{i}<\frac{1}{2}$.

Such estimates are relatively complicated to compute. Also, their dependence on nuisance parameter or nonparametric function estimates might sometimes be associated with poor finite-sample properties, indeed as the nuisance parameters here include integration orders estimates of coefficients of a reduced form of (1.1) might be expected to be worse in finite samples than in the traditional selection in which $W_{t}, U_{t}$ are treated as known to be $I(1), I(0)$, respectively. The optimal estimates of Robinson and Hualde (2003), Hualde and Robinson (2004) also depend respectively on a correct parametric specification of the short memory component of series, and smoothing numbers. Moreover the optimal estimates have to be initiated by a simple initial estimate that does not depend on estimates of the $\gamma_{i}$, at least. There is thus still interest in simpler estimates. The most obvious simple estimate is OLS. However, extending results of Robinson and Marinucci (2001) (where $P=Q=1$ ), we observe below that in some circumstances OLS converges only slowly, or not at all.

The main purpose of the present paper is to show that simple instrumental variable (IV) estimates, while still having non-standard limit distributions, can have better convergence rates than OLS, and than the narrow band least squares (NBLS) estimates of Robinson (1994), Robinson and Marinucci (2001). In such circumstances, they would thus be expected to provide better initial estimates in optimal procedures. IV estimation has previously been considered in 
a cointegration context, mostly in the traditional case of $I(1)$ observables and $I(0)$ cointegrating errors, but typically to provide representations of optimal estimates, that may also have an approximate maximum likelihood interpretation, say (see e.g. Phillips and Hansen, 1990; Kitamura and Phillips, 1995, 1997; Marmol, Escribano and Aparicio, 2002).

Our IV estimates employ only exclusion and normalization restrictions on $A$, and do not attempt to correct for cross-correlation in $U_{t}$, so it suffices to individually estimate the equations of (1.1). The following section compares rates of convergence of OLS and IV estimates of a single equation. Section 3 discusses strategies for selecting instruments. Section 4 examines finite sample performance by means of Monte Carlo simulations. Section 5 offers some concluding remarks.

\section{Instrumental Variables Estimates}

Consider the first equation of (1.1), with no loss of generality, and write it

$$
y_{t}=\beta^{\prime} x_{t}+u_{t}
$$

Here $u_{t}$ is the first element of $U_{t}, y_{t}$ is a scalar and $x_{t}$ and $\beta$ are $q \times 1$ vectors, where with no loss of generality we may take $\left(x_{t}^{\prime}, y_{t}\right)^{\prime}$ to be the leading $(q+1) \times 1$ sub-vector of $W_{t}$, for $q \leq Q$, and no element of $\beta$ is known to be zero. For brevity write $\gamma=\gamma_{1}$, and with no loss of generality set

$$
\delta_{1} \leq \delta_{2} \leq \ldots \leq \delta_{q}
$$

Thus $\left(x_{t}^{\prime}, y_{t}, u_{t}\right)$ is an $I\left(\delta_{1}, \ldots, \delta_{q+1}, \gamma\right)$ process. Note from (1.4) and (2.2) that

$$
\gamma<\delta_{q+1} \leq \delta_{1}
$$

$\delta_{q+1}$ being the integration order of $y_{t}$. Cointegration of $\left(x_{t}^{\prime}, u_{t}\right)$ is implied by (2.1) and (2.3). We have implicitly adopted the definition used, in a fractional context, by Robinson and Marinucci (2003), rather than, say, that of Johansen (1996) which requires $\gamma<\delta_{q}$.

Given a $q \times 1$ vector sequence $z_{t}, t \geq 1$, an IV estimate of $\beta$ is given by

$$
\hat{\beta}_{z}=M_{z x}^{-1} M_{z y}
$$

using the generic notation

$$
M_{a b}=\sum_{t=1}^{n} a_{t} b_{t}^{\prime}
$$

for any column vectors $a_{t}, b_{t}, t \geq 1$, and assuming $M_{z x}$ is non-singular.

We consider first the OLS special case $\hat{\beta}_{x}$. Denote by $G_{1}$ and $G_{2}$ the $q \times q$ diagonal matrices whose $i$-th diagonal elements are respectively

$$
n^{\frac{1}{2}} 1\left(\delta_{i}<\frac{1}{2}\right)+(n \log n)^{\frac{1}{2}} 1\left(\delta_{i}=\frac{1}{2}\right)+n^{\delta_{i}} 1\left(\delta_{i}>\frac{1}{2}\right)
$$


and

$n 1\left(\gamma+\delta_{i}<1\right)+(n \log n) 1\left(\gamma>0, \gamma+\delta_{i}=1\right)+n^{\gamma+\delta_{i}}\left\{1\left(\gamma+\delta_{i}>1\right)+1\left(\gamma=0, \delta_{i}=1\right)\right\}$.

If $X_{n}, n \geq 1$, and $X$ are matrices of random variables, having the same dimensions, and $g_{n}, n \geq 1$, are scalars, let $X_{n} \Rightarrow X$ denote a suitable notion of weak convergence of $X_{n}$ to $X$ as $n \rightarrow \infty$, and $X_{n} \approx g_{n}$ denote $X_{n} / g_{n} \Rightarrow X$ as $n \rightarrow \infty$. Write

$$
\delta_{*}=\min _{i: \delta_{i}>\frac{1}{2}} \delta_{i}
$$

when the right side exists, and $\beta_{i}, \hat{\beta}_{x i}$ for the $i$-th elements of $\beta, \hat{\beta}_{x}$.

Theorem 1 Let (1.4) and (2.1) hold, and

$$
G_{1}^{-1} M_{x x} G_{1}^{-1} \Rightarrow \Omega_{x x}, \quad G_{2}^{-1} M_{x u} \Rightarrow \Omega_{x u}, \text { as } n \rightarrow \infty,
$$

where $\Omega_{x x}$ is a $q \times q$ matrix that is almost surely (a.s.) finite and positive definite, and $\Omega_{x u}$ is a $q \times 1$ vector whose elements are a.s. finite. Then as $n \rightarrow \infty$, for $i=1, \ldots, q$,

$$
\begin{gathered}
\hat{\beta}_{x i}-\beta_{i} \approx 1\left(\delta_{i}<\frac{1}{2}\right)+(\log n)^{-1} 1\left(\delta_{i}=\frac{1}{2}\right)+n^{1-\delta_{i}-\delta_{*}} 1\left(\delta_{i}>\frac{1}{2}, \gamma+\delta_{*}<1\right) \\
+n^{1-\delta_{i}-\delta_{*}}(\log n) 1\left(\gamma>0, \delta_{i}>\frac{1}{2}, \gamma+\delta_{*}=1\right) \\
+n^{\gamma-\delta_{i}}\left\{1\left(\delta_{i}>\frac{1}{2}, \gamma+\delta_{*}>1\right)+1\left(\gamma=0, \delta_{*}=1\right)\right\}
\end{gathered}
$$

Theorem 1 extends the result for $\delta_{i}=1, \gamma=0$ (Stock, 1987). As in this case, the limit distribution of $\hat{\beta}_{x i}$ is non-standard and as well as depending on integration orders and other properties varies qualitatively across certain subsets of integration order space, as found by Robinson and Marinucci $(2001,2003)$. The limit distributions are in no cases normal or mixed-normal, and thus not useful to statistical inference. The proof of Theorem 1 is in the Appendix, where also sufficient conditions for (2.4), and (2.10) below, are discussed.

When $\gamma+\delta_{i}<1$, or $\gamma+\delta_{i}=1$ with $\gamma>0$, the $i$-th element of $\Omega_{x u}$ is a constant, and nonzero unless $\operatorname{cov}\left(x_{i t}, u_{t}\right)=0$, where $x_{i t}$ is the $i$-th element of $x_{t} . \Omega_{x x}$ has structure

$$
\Omega_{x x}=\left[\begin{array}{ccc}
\Omega_{x x 1} & 0 & 0 \\
0 & \Omega_{x x 2} & 0 \\
0 & 0 & \Omega_{x x 3}
\end{array}\right],
$$

where $\Omega_{x x i}$ is $q_{i} \times 1, i=1,2,3$, such that $q_{1}=\sum_{i=1}^{q} 1\left(\delta_{i}<\frac{1}{2}\right), q_{2}=\sum_{i=1}^{q} 1\left(\delta_{i}=\right.$ $\left.\frac{1}{2}\right), q_{3}=\sum_{i=1}^{q} 1\left(\delta_{i}>\frac{1}{2}\right)$. The matrices $\Omega_{x x 1}$ and $\Omega_{x x 2}$ are constant, and positive definite. For $i$ such that $\gamma+\delta_{i}=1$ and $\delta_{i}=\frac{1}{2}$ the constant and nonzero/positive definite properties result, despite the nonstationarity, from domination of the 
standard deviation by the mean, see Theorems 4.2 and 5.1 of Robinson and Marinucci (2001). The block-diagonal structure in (2.6) does not require any assumptions of orthogonality between elements of $x_{t}$, but rather is due to the differing normalisations. The block-diagonality ensures consistency of $\hat{\beta}_{x i}$ for $i>q_{1}$, despite the inconsistency of $\hat{\beta}_{x i}$ for $i \leq q_{1}$ (which is due to simultaneous equations bias). For $x_{i t}$ on the stationary-nonstationary boundary $\hat{\beta}_{x i}$ is $\log n$ consistent. For nonstationary $x_{i t}$ with $\delta_{i}>\frac{1}{2}$, we obtain the "optimal" $n^{\delta_{i}-\gamma_{-}}$ consistency of $\hat{\beta}_{x i}$ when

$$
\gamma+\delta_{j}>1 \text { or } \gamma=0, \delta_{j}=1
$$

for all $j$ such that $\delta_{j}>\frac{1}{2}$, but otherwise the rate of $\hat{\beta}_{x i}$ is restricted: not only if $\gamma+\delta_{i}<1$ or $\gamma+\delta_{i}=1$ with $\gamma>0$, but also if $\gamma+\delta_{i}>1$ and there is a single $\delta_{j}>\frac{1}{2}$ such that $\gamma+\delta_{j}<1$ or $\gamma+\delta_{j}=1$ with $\gamma>0$. The rates in (2.5) seem sharp, except in the event of constant elements of $\Omega_{x x}$ or $\Omega_{x u}$ taking particular values.

No rate improvement in (2.5) seems possible if $\delta_{i}>\frac{1}{2}$ and $\gamma+\delta_{i}>1$ or $\gamma=0$ with $\delta_{i}=1$ for all $i$, which includes the traditional case of $I(1)$ regressors and $I(0)$ disturbances. However, while it is common practice to test the $I(1)$ assumption, and it is frequently not rejected, the tests commonly used, such as Dickey-Fuller, do not have particularly good power against fractional alternatives. It may not be possible to rule out the presence of mean-reverting, or even stationary, $x_{i t}$, and visual discrimination based on the observed trajectory can be hazardous. Improvements to OLS are possible when for some $i, \gamma+\delta_{i}=1$ with $\gamma>0$ or $\gamma+\delta_{i}<1$, and the latter inequality always holds when $x_{i t}$ is stationary or when $x_{i t}$ is nonstationary and mean-reverting but $\gamma=0$ (and also for sufficiently small positive $\gamma)$. To guard against such possibilities we consider the IV estimate $\hat{\beta}_{z}$ with $z_{t}$ an $I\left(\xi_{1}, \ldots, \xi_{q}\right)$ process, with

$$
\xi_{1} \leq \xi_{2} \leq \ldots \leq \xi_{q} .
$$

For simplicity we consider only cases in which

$$
\delta_{i}+\xi_{i}>1, \quad i=1, \ldots, q .
$$

Denote by $G_{3}, G_{4}$ and $G_{5}$ the $q \times q$ diagonal matrices with $i$-th diagonal elements $n^{\xi_{i}}, n^{\delta_{i}}$ and

$n 1\left(\gamma+\xi_{i}<1\right)+(n \log n) 1\left(\gamma>0, \gamma+\xi_{i}=1\right)+n^{\gamma+\xi_{i}}\left\{1\left(\gamma+\xi_{i}>1\right)+1\left(\gamma=0, \xi_{i}=1\right)\right\}$ and by $\hat{\beta}_{z i}$ the $i$-th element of $\hat{\beta}_{z}$.

Theorem 2 Let (1.4) and (2.1) hold, and

$$
G_{3}^{-1} M_{z x} G_{4}^{-1} \Rightarrow \Omega_{z x}, \quad G_{5}^{-1} M_{z u} \Rightarrow \Omega_{z u},
$$

where $\Omega_{z x}$ is an a.s. finite and non-singular matrix and $\Omega_{z u}$ is an a.s. finite vector. Then as $n \rightarrow \infty$, for $i=1, \ldots, q$,

$$
\begin{aligned}
\hat{\beta}_{z i}-\beta_{i} \approx & n^{1-\delta_{i}-\xi_{1}} 1\left(\xi_{1}+\gamma<1\right)+n^{1-\delta_{i}-\xi_{1}}(\log n) 1\left(\gamma>0, \xi_{1}+\gamma=1\right) \\
& +n^{\gamma-\delta_{i}}\left\{1\left(\xi_{1}+\gamma>1\right)+1\left(\gamma=0, \xi_{1}=1\right)\right\} .
\end{aligned}
$$


The proof is again left to the Appendix. Again, limit distributions are in general non-standard and depend on integration orders and other features, and vary qualitatively across regions of integration order space, as well as with other properties of the instruments $z_{i}$. Thus again limit distributions will not be useful in inference, and we have not felt it worthwhile to take up space representing them.

The $i$-th element of $\Omega_{z u}$ is constant when $\gamma+\xi_{i} \leq 1$,or when $\gamma+\xi_{i}=1$ with $\gamma>0$. In view of (2.8) $\hat{\beta}_{z i}$ is consistent even in case of stationary $x_{i t}$, while the optimal rate $n^{\gamma-\delta_{i}}$ is achieved for all $i$ when

$$
\xi_{1}+\gamma>1 \text { or } \gamma=0, \xi_{1}=1 \text {, }
$$

which for $\xi_{1}>\delta_{*}$ is a milder condition than $\delta_{*}+\gamma>1$ or $\gamma=0, \delta_{*}=1$ (cf. (2.5)). The dependence of the right side on $\xi_{1}$, rather than $\xi_{i}$, due to the general non-diagonality of $\Omega_{z x}$.

The NBLS estimate proposed by Robinson (1994) to consistently estimate cointegrating relations with stationary $I(d)$ regressors was also shown by Robinson and Marinucci (2001) to improve convergence rates in the sort of nonstationary environments in which our IV approach provides improvements over OLS. NBLS also has an IV interpretation and is intuitively appealing, but its convergence rate (which depends on the rate of a bandwidth number as $n \rightarrow \infty$ ) is not necessarily better or worse than that of our simple IV estimate $\hat{\beta}_{z}$ in the stationary regressor case, and it is worse in the nonstationary case when $\gamma+\delta_{i}<1$ or $\gamma+\delta_{i}=1$ with $\gamma>0$ for some $i$, but (2.12) holds. Instead of the arbitrariness of bandwidth choice in the NBLS approach, $\hat{\beta}_{z}$ suffers from arbitrariness of instrument, but it is computationally simpler, and applied economists may feel more comfortable with its familiar form.

\section{Choice of Instrument}

In classical, stable environments, instruments are ideally chosen to be orthogonal to disturbances, but highly correlated with the variables they replace. In the unit root cointegration literature this prescription does not apply : instruments and can be correlated with disturbances, and independent of regressors (see, e.g. Phillips, 1986, Phillips and Hansen, 1990). The latter situation is possible in our more general setting, when $\delta_{i}>\frac{1}{2}, \xi>\frac{1}{2}$ for all $i$. We identify below several strategies for choosing instruments. All the estimates covered in Theorem 2 are sub-optimal, and it does not seem possible to find a statistically best choice of instrument $z_{t}$. Thus there is inevitably considerable ambiguity in the choice of $z_{t}$, and our discussion illustrates this, while also identifying other advantages, or disadvantages, of particular ones. In principle the number of possible instruments one might use is limitless, especially as one could use linear combinations of ones suggested below, or lagged versions, or IV versions of the narrow-band estimate (4.1) employed in the Monte Carlo study of the following section. 
1. Cointegration in (2.1) requires that at least two elements of $\left(x_{t}^{\prime}, y_{t}\right)$ have the same integration order. In the special case when this is true of all elements, convergence rates are constant across $\hat{\beta}_{x}$. There may be other elements of $W_{t}$ with different integration orders. Robinson and Yajima (2002) investigated "simple cointegration", in which (1.1) consists of subsystems involving non-overlapping elements of $W_{t}$, integration orders being constant within, but not across, sub-systems. Then if they satisfy (2.9), variables in one system might be suitable instruments in another sub-system that involves variables of lower integration order. Robinson and Yajima (2002) provided an algorithm for partitioning $W_{t}$ into subsets with common integration orders, and thence determining the cointegrating rank of each sub-system.

2. Evidently this approach cannot generate instruments for each equation of (1.1), and uncertainty about integration orders of observables presents some ambiguity. A simple approach is to integrate observables, possibly $x_{t}$ in (2.1). For simplicity take $x_{t}$ to be scalar, and define, for any sequence $r_{t}, r_{t}^{(\alpha)}=\Delta^{-\alpha} r_{t}^{\#}, \alpha>0$. Then if $x_{t}$ is $I(\delta), x_{t}^{(\alpha)}$ is $I(\delta+\alpha)$. We might thus choose $z_{t}=x_{t}^{(\alpha)}$ as an instrument, for suitable $\alpha$. If it is believed that $\delta>\frac{1}{2}$, then $\alpha_{i}=\frac{1}{2}$ suffices for (2.9), whereas $\alpha=1$ suffices for any $\delta>0$. Clearly $\alpha=1$ is an attractively simple option.

3. A similar outcome is achieved by instead integrating the model (2.1). Taking $q=1$ again, and forming

$$
y_{t}^{(\alpha)}=\beta x_{t}^{(\alpha)}+u_{t}^{(\alpha)},
$$

an IV estimate using $x_{t}$ as instrument for $x_{t}^{(\alpha)}$ can be written $\tilde{\beta}=\sum_{t=1}^{n} x_{t} y_{t}^{(\alpha)} / \sum_{t=1}^{n} x_{t} x_{t}^{(\alpha)}$. In view of $(1.2) \tilde{\beta}=x^{\prime} B^{(\alpha)} y / x^{\prime} B^{(\alpha)} x$, where $x=\left(x_{1}, \ldots, x_{n}\right)^{\prime}, y=$ $\left(y_{1}, \ldots, y_{n}\right)^{\prime}$ and $B^{(\alpha)}$ is the $n \times n$ lower-triangular matrix whose $t$-th row is $\left(a_{0}(\alpha), a_{1}(\alpha), \ldots, a_{t-1}(\alpha), 0, \ldots, 0\right)$. But this is also an IV estimate for (2.1) when the instrument for $x_{t}$ is the $t$-th element of $B^{(\alpha) \prime} x$. On the other hand the IV estimate for (2.1) that uses $x_{t}^{(\alpha)}$ as instrument for $x_{t}$ is $\hat{\beta}_{x^{(\alpha)}}=x^{\prime} B^{(\alpha) \prime} y / x^{\prime} B^{(\alpha) \prime} x$. Clearly $\tilde{\beta}$ and $\hat{\beta}_{x^{(\alpha)}}$ have the same convergence rates.

4. It would be possible to generalize our definition of $W_{t}$ to allow for deterministic effects, in particular each element could be a sum of an $I(d)$ variable and a sequence asymptotic to $t^{c}$, for some $c$, where either stochastic or deterministic components may dominate asymptotically. This suggests using increasing functions of $t$ as instruments. But this can work also for purely stochastic nonstationary $z_{t}$, due to a "spurious regression" effect (Phillips, 1988). For example with $q=1$, using $z_{t}=t^{\xi-\frac{1}{2}}$ for $\xi>\frac{1}{2}$ produces the same convergence rate as an $I(\xi) z_{t}$ (albeit a different type of limit distribution, possibly normal). 
5. The "spurious regression" phenomenon also suggests artificial generation of instruments. In particular, for nonstationary $x_{i t}$, an $I\left(\xi_{i}\right)$ instrument of the form $\Delta^{-\xi_{i}} e_{t}^{\#}$ would suffice, where $\xi_{i}>\frac{1}{2}$ and $e_{1}, e_{2}, \ldots$, is a sequence from a Monte Carlo random number generator. Many workers would resist such a device, however, in part because calculations would be difficult to replicate and empirical findings liable to be greeted with suspicion.

\section{Finite Sample Behaviour}

Our IV proposals are based on consideration of asymptotic rates of convergence, so it is important to investigate performance in finite samples. We focus only on moderate series lengths $n$, to gauge relevance to macroeconomic analysis. For long financial series our convergence rates would be more directly informative, and simulation evidence of less importance. We compare some of the choices of instrument described in the previous section, including also OLS, as well as NBLS. The latter, when $q=1$ in $(2.1)$, is

$$
\tilde{\beta}_{m}=\left(\sum_{j=1}^{m}\left|w_{x}\left(\lambda_{j}\right)\right|^{2}\right)^{-1} \operatorname{Re}\left\{\sum_{j=1}^{m} w_{x}\left(\lambda_{j}\right) w_{y}\left(-\lambda_{j}\right)\right\},
$$

where

$$
w_{a}(\lambda)=(2 \pi n)^{-\frac{1}{2}} \sum_{t=1}^{n} a_{t} e^{i t \lambda}
$$

and $\lambda_{j}=2 \pi j / n, 1 \leq m<n / 2$. The integer $m$ is chosen by the practitioner: for given $n$, bias tends to increase, and variance to decrease, with $m$. If $\left(x_{t}, u_{t}\right)$ is an $I(\delta, \gamma)$ process with $\gamma<\delta$ (cf. (1.4)), then under regularity conditions and with $m \rightarrow \infty, m / n \rightarrow 0$ as $n \rightarrow \infty, \tilde{\beta}_{m}$ is consistent for $\beta$, and moreover

$$
\begin{aligned}
\tilde{\beta}_{m}-\beta & \approx(n / m)^{\gamma-\delta} 1\left(\delta<\frac{1}{2}\right)+n^{\gamma-\delta} m^{1-\gamma-\delta} 1\left(\delta>\frac{1}{2}, \gamma+\delta<1\right) \\
+n^{-1} 1(\gamma & =0, \delta=1)+(\log n / n) 1(\gamma>0, \gamma+\delta=1)+n^{\gamma-\delta} 1(\gamma+\delta>1)
\end{aligned}
$$

(see Robinson, 1994; Robinson and Marinucci 2001). These authors also characterized limit distributions when $x_{t}$ is nonstationary, and Christensen and Nielsen (2004) recently established asymptotic normality in the stationary case, when also $\gamma+\delta<1 / 2$ and $x_{t}$ and $u_{t}$ are incoherent at frequency zero. Unlike OLS, NBLS is consistent in case of stationary $x_{t}$, whereas for nonstationary $x_{t}$ NBLS converges faster than OLS when $\gamma+\delta<1$ or $\gamma>0, \gamma+\delta=1$, but slower than IV given a suitable choice of instrument (see (2.5), (2.11)). NBLS has been employed in a number of empirical investigations (see e.g. Bandi and Perron, 2004; Christensen and Nielsen, 2004; Robinson and Marinucci, 2003), so it seems appropriate to see how it compares with IV in our numerical study.

Except in our final experiment, all $I(d)$ time series generated were of basic fractional type with $\operatorname{NID}(0,1) I(0)$ source sequence. We allowed for various 
integration orders and levels of cross-correlation between series, and compared Monte Carlo bias and standard deviation (SD) across 2000 replications of various estimates computed from $n=64$ and $n=128$ observations.

Our first version of (2.1) took $q=1$, with $\beta=2,\left(u_{t}, x_{t}\right) \sim I(\gamma, \delta)$. As well as computing OLS $\left(\hat{\beta}_{x}\right)$ and $\mathrm{NB}_{4}$ and $\mathrm{NB}_{5}$ (where $\mathrm{NB}_{m}$ is $\tilde{\beta}_{m}(4.1)$ ), we computed three versions of IV $\hat{\beta}_{z}$, denoted $\operatorname{IV}_{w}$ and $\operatorname{IV}_{(\alpha)}$ for $\alpha=0.5,1$. $\mathrm{IV}_{w}$ uses $z_{t}=w_{t} \sim I(\chi)$ under the assumption that $W_{t}$ in (1.1) includes an additional variable $w_{t} ; \mathrm{IV}_{(\alpha)}$ takes $z_{t}=x_{t}^{(\alpha)}$. Thus, $\mathrm{IV}_{w}, \mathrm{IV}_{(.5)}$ and $\mathrm{IV}_{(1)}$ entail $\xi=\chi, \delta+0.5$ and $\delta+1$, respectively. We considered four choices of $(\gamma, \delta, \chi)$. In case (a) $(\gamma, \delta, \chi)=(0.1,0.3,0.7)$. Here, $x_{t}$ is stationary, $w_{t}$ satisfies $\delta+\xi=1$ and $\gamma+\xi<1$ (cf. (2.9), (2.12)), $x_{t}^{(.5)}$ satisfies (2.9) and $\gamma+\xi<1$ (cf. (2.12)), and $x_{t}^{(1)}$ satisfies $(2.9)$ and $(2.12)$. In case $(\mathrm{b}),(\gamma, \delta, \chi)=(0.2,0.6,0.9)$. Here, $x_{t}$ is nonstationary but satisfies $\gamma+\delta<1$ (cf. (2.7)) while all choices of $z_{t}$ satisfy (2.9) and (2.12). In case $(\mathrm{c})(\gamma, \delta, \chi)=(0.4,0.7,0.8)$. Here, $(2.9),(2.9)$ and $(2.12)$ are satisfied. The same is true in case $(\mathrm{d}),(\gamma, \delta, \chi)=(0,1,1)$, which covers the usual $I(0) / I(1)$ situation. Convergence rates of the various estimates are displayed in Table 1.

(Table 1 about here)

We also varied the cross-correlation structure of the $\operatorname{NID}(0,1)$ innovations of $u_{t}, x_{t}, w_{t}$, denoted $v_{u t}, v_{x t}, v_{w t}$, respectively. In case (i) we took $v_{u t}, v_{x t}, v_{w t}$ to be mutually independent, so that OLS is consistent even when $x_{t}$ is stationary and $w_{t}$ is "spurious". In cases (ii)-(iv), $\operatorname{cov}\left(v_{u t}, v_{w t}\right)=\operatorname{cov}\left(v_{x t}, v_{w t}\right)=0.5$ throughout, but we varied $\rho=\operatorname{cov}\left(v_{u t}, v_{x t}\right)$ taking $\rho=0.5,0.7,0.9$, respectively. These four cases are indicated in Tables 2 and 3 by the $\rho$ column, where the different treatment of other correlations in case (i) must be borne in mind.

(Table 2 about here)

Table 2 presents Monte Carlo results when $n=64$. In case (a), where $x_{t}$ is stationary, OLS unsurprisingly performs increasingly poorly as $\rho$ increases, with respect to bias as well as $\mathrm{SD}$. $\mathrm{IV}_{w}$ registers considerable improvement, though $\mathrm{IV}_{(\alpha)}$ does better, especially when $\alpha=1$, the results reflecting the ordering of rates in Table 1 . However, the $\mathrm{NB}_{m}$ are clearly superior, indicating an advantage in this case for those computationally more complex estimates. The pattern as $\rho$ increases across cases (ii)-(iv) is not very clear where the IV and NBLS estimates are concerned: sometimes the best results are found when $\rho=0.9$. In case (b) the rather narrow inferiority of OLS seen in Table 1 is borne out in the Monte Carlo results. Generally in cases (b)-(d) there is not much to choose between NBLS and IV, with the latter sometimes superior, but in cases (b) and (c) the same ordering of IV estimates as in case (a) is found. Notice that SD can be affected by choice of $z_{t}$ even when convergence rate is not, and this may explain why $\mathrm{IV}_{w}$ is less variable than OLS even in case (d). Table 3 contains corresponding results for $n=128$ The fact that the bias of OLS is not reduced is unsurprising. Bias is otherwise on the whole somewhat reduced, as is $\mathrm{SD}$, except for the NBLS estimates; this reflects the fact that under suitable conditions the asymptotic variance of $\mathrm{NB}_{m}$ is of order $m^{-1}$. We also obtained results with $z_{t}=x_{t}^{(2)}$ and some deterministic $z_{t}$; these are unreported because 
in the former case they differed little from those for $z_{t}=x_{t}^{(1)}$, and in the latter case because they were systematically worse than those for the stochastic $z_{t}$.

(Table 3 about here)

We also investigated effects of differential integration orders in multiple regression models. We first took $q=3$ in (2.1), $\beta_{1}=2.5, \beta_{2}=2, \beta_{3}=1.5$, and $\gamma=0.1, \delta_{1}=0.4, \delta_{2}=0.7, \delta_{3}=1$. Thus we have a stationary regressor, a nonstationary mean-reverting one and a unit root one. We considered four different covariance structures for the $\operatorname{NID}(0,1)$ innovations $v_{u t}, v_{i t}$ of $u_{t}$ and $x_{i t}, i=1,2,3$. Using the notation $\rho_{i}=\operatorname{cov}\left(v_{u t}, v_{i t}\right), \rho_{i j}=\operatorname{cov}\left(v_{i t}, v_{j t}\right)$, these are as follows:

Case (I): $\rho_{i}=\rho_{12}=\rho_{13}=0, \quad i=1,2,3 ; \quad \rho_{23}=0.5$.

Case (II): $\rho_{i}=\rho_{i j}=0.5, \quad i=1,2,3, \quad j \neq i$.

Case (III): $\rho_{2}=\rho_{3}=\rho_{i j}=0.5, \quad i=1,2,3, j \neq i ; \rho_{1}=0.7$.

Case (IV): $\rho_{2}=\rho_{3}=\rho_{i j}=0.5, \quad i=1,2,3, j \neq i ; \rho_{1}=0.9$.

In case (I) there is correlation only between the nonstationary regressors $x_{2 t}$ and $x_{3 t}$, whereas in the other three cases all innovation correlations are 0.5 except for that between $v_{u t}$ and $v_{1 t}$, which varies.

(Table 4 about here)

Table 4 contains rates of convergence of OLS and $\operatorname{IV}_{(\alpha)}, \alpha=0.5,1$, where $\operatorname{IV}_{(\alpha)}$ is $\hat{\beta}_{z}$ with $z_{t}=\left(x_{1 t}^{(\alpha)}, x_{2 t}^{(\alpha)}, x_{3 t}^{(\alpha)}\right)^{\prime}: \operatorname{IV}_{(.5)}$ overcomes the inconsistency in the OLS estimate of $\beta_{1}$, and improves on the rates of the OLS estimates of $\beta_{2}$ and $\beta_{3}$, while a small further improvement is registered by $\operatorname{IV}_{(1)}$. Tables 5-7 compare finite sample performance of these estimates, each table including results for both $n=64$ and 128, and the four increasing values of $\rho_{1}$ indexing cases (I)-(IV). Apart from the poor performance of the OLS estimate of $\beta_{1}$ in case (I), the results seem quite consistent with the asymptotics. In case (I) OLS of $\beta_{2}$ and $\beta_{3}$ does well, but in the other cases $\operatorname{IV}_{(.5)}$ is better and $\operatorname{IV}_{(1)}$ better still, perhaps more than one might anticipate from Table 4. More surprising is the systematic way in which SD falls from OLS through $\mathrm{IV}_{(.5)}$ to $\mathrm{IV}_{(1)}$.

(Tables 5-7 about here)

Our next experiment focussed more explicitly on the curse-of-dimensionality dangers of multiple regression, taking $q=8$ in (2.1). To keep the design simple, the elements of $x_{t}$ were mutually independent, and $I(\delta)$ for the same $\delta$ and the same scale, and they were independent also of $u_{t}$, which was $I(\gamma)$. We took $\beta=(2,2.5,1.5,3,3.5,1,4,8)^{T}$, and employed three $(\gamma, \delta)$ combinations. As in the previous experiment, we compared OLS, $\mathrm{IV}_{(.5)}$ and $\mathrm{IV}_{(1)}$. As expected the results are similar across the elements of the $\beta$ estimates, albeit with some variation in the direction of bias, and we report in Tables 8 and 9 only results for the first two elements, which seem to exhibit the bias variation. OLS is seriously biased for the stationary case $(\gamma, \delta)=(0.1,0.4)$, despite the independence between $x_{t}$ and $u_{t}$ (as found in previous experiments) with $\mathrm{IV}_{(.5)}$ and $\mathrm{IV}_{(1)}$ making considerable improvements; they also significantly reduce SD. For the other $(\gamma, \delta)$ combinations OLS performs reasonably, but the IV estimates do 
somewhat better. In all cases there is improvement with increasing $n$. On the whole, $\mathrm{IV}_{(1)}$ does slightly better than $\operatorname{IV}_{(.5)}$.

(Tables 8-9 about here)

Spurious regression was mentioned in the preceding section. This was originally identified as an issue in case of regression between independent $I(1)$ processes. More recently, Cappuccio and Lubian (1997), Tsay and Chung (2000) have studied its effects in the context of stationary and nonstationary fractional processes, focussing on the behaviour of OLS regression estimates (as well as more basic statistics, and OLS-based statistics such as $t$-ratios). We compare our IV estimates with OLS and NBLS. The model is (2.1) with $q=1$ and $\beta=0$, and $x_{t}$ and $y_{t}=u_{t}$ are independent $I(\delta)$ processes. (Thus, this is actually not a cointegrated model.) In Table $10 \mathrm{IV}_{w}$ uses an instrument $w_{t}$ that is $I(\chi)$; the $(\delta, \chi)$ combinations are $\left(\mathrm{a}^{\prime}\right):(.3, .7) ;\left(\mathrm{b}^{\prime}\right):(.6, .8) ;\left(\mathrm{c}^{\prime}\right):(.7, .9)$; and $\left(\mathrm{d}^{\prime}\right):(1,1)$. Spurious effects are, as expected, greatest in the "most nonstationary" unit root case $\left(\mathrm{d}^{\prime}\right)$, and we find that NBLS and our IV estimates manage to noticeably reduce them, with NBLS doing best, followed by the $\mathrm{IV}_{(\alpha)}$ estimates. The same general pattern is repeated for the other parameter values, with both bias and SD decreasing as $\delta$ decreases. Throughout there is some improvement with increasing $n$.

(Table 10 about here)

Our final experiment partially relaxes the Gaussian prescription by allowing the disturbances $u_{t}$ to have innovations from a $t_{5}$ distribution, so they have finite moment of order 4 only. One expects deterioration of OLS estimates in the presence of fat tails, and we wish to examine how the IV and NBLS estimates compare. The experiment is also designed so as to allow comparisons with Tables 2 and 3: we have $q=1, \beta=2$, and take the same $(\gamma, \delta, \xi)$ combinations as there, though we now only consider the correlation $\rho=0.5$ between $u_{t}$ and $x_{t}$. There is little difference in the biases from those of Tables 2 and 3, but unsurprisingly the standard deviations increase. Except when $(\gamma, \delta, \chi)=$ $(0,1,1)$, OLS clearly performs worst and the two versions of NBLS narrowly outperform the IV estimates.

\section{Final Comments}

We have shown that IV estimates of cointegrating relations can improve on OLS when the latter falls short of optimal rate. Our fractional integration setting illustrates much of the scope of the approach but is not essential, the methods applying also in the more traditional case of integer orders: to guard against the possibility that a regressor is $I(0)$ rather than $I(1)$ (or $I(1)$ rather than $I(2)$ ) an $I(1)$ (or $I(2)$ ) instrument might be employed. Several choices of instrument are proposed, including a variable that appears elsewhere in the system, and fractional or integer integration of the regressor. In a Monte Carlo study, NBLS estimates, employed in a number of recent empirical studies, sometimes perform better, but perhaps not sufficiently to offset the advantages of simplicity and 
familiarity of IV. Of the instruments employed, the simple and always-available one of partially summing the regressor seems generallly to perform best.

\section{Appendix}

\section{Proof of Theorem 1: Write}

$$
\hat{\beta}_{x}-\beta=G_{1}^{-1}\left(G_{1}^{-1} M_{x x} G_{1}^{-1}\right)^{-1} G_{1}^{-1} G_{2}\left(G_{2}^{-1} M_{x u}\right) .
$$

From (2.4) this is dominated as $n \rightarrow \infty$ by $G_{1}^{-1} \Omega_{x x}^{-1} G_{1}^{-1} G_{2} \Omega_{x u}$. Now $G_{1}^{-1} G_{2}$ has $i$-th diagonal element

$$
\begin{aligned}
n^{\frac{1}{2}} 1\left(\delta_{i}\right. & \left.<\frac{1}{2}\right)+(n / \log n)^{\frac{1}{2}} 1\left(\delta_{i}=\frac{1}{2}\right)+n^{1-\delta_{i}} 1\left(\delta_{i}>\frac{1}{2}, \gamma+\delta_{i}<1\right) \\
+n^{1-\delta_{i}}(\log n) 1(\gamma & \left.>0, \delta_{i}=1\right)+n^{\gamma}\left\{1\left(\gamma+\delta_{i}>1\right)+1\left(\gamma=0, \delta_{i}=1\right)\right\} .
\end{aligned}
$$

By the block-diagonality of $\Omega_{x x}, \hat{\beta}_{x i}-\beta_{i}=O_{p}(1)$ for $i=1, \ldots, q_{1}$, and $\hat{\beta}_{x i}-\beta_{i}=$ $O_{p}\left((\log n)^{-1}\right)$ for $i=q_{1}+1, \ldots, q_{1}+q_{2}$. In general $\Omega_{x x 3}$ is not a diagonal matrix. Denoting by $H$ the matrix consisting of the last $q_{3}$ rows and columns of $G_{1}^{-1} G_{2}$, a typical element $c_{i j}$ of $\Omega_{x x 3}^{-1} H$ satisfies

$$
\begin{gathered}
c_{i j} \approx n^{1-\delta_{*}} 1\left(\gamma+\delta_{*}<1\right)+n^{1-\delta_{*}}(\log n) 1\left(\gamma>0, \gamma+\delta_{*}=1\right) \\
\left.+n^{\gamma}\left\{1\left(\gamma+\delta_{*}>1\right)+1\left(\gamma=0, \delta_{*}=1\right)\right\}\right), i, j>q_{1}+q_{2} .
\end{gathered}
$$

The proof is now readily completed.

Proof of Theorem 2: Write

$$
\hat{\beta}_{z}-\beta=G_{4}^{-1}\left(G_{3}^{-1} M_{z x} G_{4}^{-1}\right)^{-1} G_{3}^{-1} G_{5}\left(G_{5}^{-1} M_{z u}\right) .
$$

By (2.10) this is dominated by $G_{4}^{-1} \Omega_{z x}^{-1} G_{3}^{-1} G_{5} \Omega_{z u}$ as $n \rightarrow \infty$. The $i$-th diagonal element of $G_{3}^{-1} G_{5}$ is

$$
\begin{aligned}
& n^{1-\xi_{i}} 1\left(\gamma+\xi_{i}<1\right)+n^{1-\xi_{i}}(\log n) 1\left(\gamma>0, \gamma+\xi_{i}=1\right) \\
& +n^{\gamma}\left\{1\left(\gamma+\xi_{i}>1\right)+1\left(\gamma=0, \xi_{i}=1\right)\right\} .
\end{aligned}
$$

Bearing in mind the general non-diagonality of $\Omega_{z x}$, and (2.8), the proof is readily completed.

Sufficient conditions for (2.4) and (2.10): We need to discuss the underlying $\overline{I(0)}$ vector generating $x_{t}, y_{t}$ and, when it is involved, $z_{t}$ (bearing in mind (1.3) and that $z_{t}$ may have a common or overlapping $I(0)$ source with $x_{t}$ and $\left.y_{t}\right)$. Denote by $v_{t}$ this $I(0)$ vector, and assume that

$$
v_{t}=\mu+\sum_{j=-\infty}^{\infty} A_{j} \varepsilon_{t-j}, \quad \sum_{j=0}^{\infty} \sum_{k=j+1}^{\infty}\left\{\left\|A_{k}\right\|^{2}+\left\|A_{-k}\right\|^{2}\right\}<\infty
$$


where the $A_{j}$ are square matrices, the $\varepsilon_{t}$ are iid vectors with zero mean, covariance matrix $\Omega$ and finite moments of all orders, $\sum_{j=-\infty}^{\infty} A_{j}$ and $\Omega$ are of full rank, and $\mu$ is a mean vector. These conditions are sufficient for (2.4) and (2.10). They are designed principally to utilize results of Marinucci and Robinson (2000) (see also Akonom and Gourieroux, 1987) to the extent that convergence of fractional Brownian motion of $x_{t}, u_{t}$ or $z_{t}$ underlies (2.4) and (2.10), but they also imply conditions for Theorems 4.1-4.5 and Theorem 5.1 of Robinson and Marinucci (2001), which (2.4) and (2.10) also use. They easily cover Gaussian ARMA $v_{t}$.

\section{References}

Akonom, J. \& C. Gourieroux (1987) A functional central limit theorem for fractional processes. Preprint.

Bandi, F. \& B. Perron (2004) Long memory and the relation between implied and realized volatility. Preprint.

Cappuccio, N. \& D. Lubian (1997) Spurious regressions between $I(1)$ processes with long memory errors. Journal of Time Series Analysis 18, 341-354.

Cheung, Y.W. \& K.S. Lai (1993) A fractional cointegration analysis of purchasing power parity. Journal of Business and Economic Statistics 11, 103-112.

Christensen, B.J. \& M.O. Nielsen (2004) Asymptotic normality of narrowband least squares in the stationary fractional cointegration model and volatility forecasting. Journal of Econometrics, forthcoming.

Dolado, J. \& F. Marmol (1997) Efficient estimation of cointegrating relationships among higher order and fractionally integrated processes. Preprint.

Hualde, J. \& P.M. Robinson (2004) Semiparametric estimation of fractional cointegration. Preprint.

Hualde, J. \& P.M. Robinson (2006) Root-n-consistent estimation of weak fractional cointegration. Journal of Econometrics, forthcoming.

Jeganathan, P. (1999) On asymptotic inference in cointegrated time series with fractionally integrated errors. Econometric Theory 15, 583-621.

Johansen, S. (1991) Estimation and hypothesis testing of cointegrating vectors in Gaussian vector autoregressive models. Econometrica 59, 1551-1580.

Johansen, S. (1996) Likelihood-Based Inference in Cointegrated Vector Autoregressive Models. Oxford: Oxford University Press.

Kim, C.S. \& P.C.B. Phillips (2000) Fully modified estimation of fractional cointegration models. Preprint.

Kitamura, Y. \& P.C.B. Phillips (1995) Efficient IV estimation in nonstationary regression: an overview and simulation study. Econometric Theory 11, 1095-1130.

Kitamura, Y. \& P.C.B. Phillips (1997) Fully modified IV, GIVE and GMM estimation with possibly non-stationary regressors and instruments. Journal of Econometrics 80, 85-123. 
Marinucci, D. \& P.M. Robinson (2000) Weak convergence of multivariate fractional processes. Stochastic Processes and their Applications 86, 103-120.

Marmol, F., A. Escribano \& F.M. Aparicio (2002) Instrumental variable interpretation of cointegration with inference results for fractional cointegration. Econometric Theory 18, 646-672.

Phillips, P.C.B. (1986) Understanding spurious regressions in econometrics. Journal of Econometrics 33, 311-340.

Phillips, P.C.B. (1991) Optimal inference in cointegrated systems. Econometrics 59, 283-306.

Phillips, P.C.B. \& B.E. Hansen (1990) Statistical inference in instrumental variables regression with I(1) processes. Review of Economic Studies 57, 99-125.

Robinson, P.M. (1994) Semiparametric analysis of long memory time series. Annals of Statistics 22, 515-539.

Robinson, P.M. \& J. Hualde (2003) Cointegration in fractional systems with unknown integration orders. Econometrica 71, 1727-1766.

Robinson, P.M. \& D. Marinucci (2001) Narrow band analysis of nonstationary processes. Annals of Statistics 29, 947-986.

Robinson, P.M. \& D. Marinucci (2003) Semiparametric frequency domain analysis of fractional cointegration. In P.M. Robinson (ed.) Time Series with Long Memory. Oxford: Oxford University Press, pp. 334-373.

Robinson, P.M. \& Y. Yajima (2002) Determination of cointegrating rank in fractional systems. Journal of Econometrics 106, 217-241.

Stock, J. (1987) Asymptotic properties of least squares estimators of cointegrating vectors. Econometrica 58, 1035-1056.

Stock, J. \& M.W. Watson (1988) Testing for common trends. Journal of the American Statistical Society 83, 1097-1107.

Tsay, W.-J. \& C.-F. Chung (2000) The spurious regression of fractionally integrated processes. Journal of Econometrics 96, 155-182. 
Table 1: Convergence rates of deviations of estimates from $\beta$ in model (2.1) with $q=1$ in cases (I)-(IV). $X=$ "inconsistent".

\begin{tabular}{|c|c|c|c|c|c|}
\hline$\gamma, \delta, \chi$ & OLS & $\mathrm{NB}_{m}$ & $\operatorname{IV}_{w}$ & $\operatorname{IV}_{(.5)}$ & $\operatorname{IV}_{(1)}$ \\
\hline $.1, .3, .7$ & $X$ & $(m / n)^{-2}$ & $(\log n)^{-1}$ & $n^{-.1}$ & $n^{-.2}$ \\
$.2, .6, .9$ & $n^{-.2}$ & $m^{2} n^{-.4}$ & $n^{-.4}$ & $n^{-.4}$ & $n^{-.4}$ \\
$.4, .7, .8$ & $n^{-.3}$ & $n^{-.3}$ & $n^{-.3}$ & $n^{-.3}$ & $n^{-.3}$ \\
$0,1,1$ & $n^{-1}$ & $n^{-1}$ & $n^{-1}$ & $n^{-1}$ & $n^{-1}$ \\
\hline
\end{tabular}

Table 2: Monte Carlo bias and SD of estimates of $\beta$ in model (2.1) with $q=1$ in cases (a)-(d) and (i)-(iv), with $n=64$, and 2000 replications

\begin{tabular}{|c|c|c|c|c|c|c|c|c|c|c|c|c|c|}
\hline & & \multicolumn{6}{|c|}{ Bias } & \multicolumn{6}{|c|}{ SD } \\
\hline$\gamma, \delta, \chi$ & $\rho$ & OLS & $\mathrm{NB}_{4}$ & $\mathrm{NB}_{5}$ & $\mathrm{IV}_{w}$ & $\operatorname{IV}_{(.5)}$ & $\operatorname{IV}_{(1)}$ & OLS & $\mathrm{NB}_{4}$ & $\mathrm{NB}_{5}$ & $\mathrm{IV}_{w}$ & $\operatorname{IV}_{(.5)}$ & $\mathrm{IV}_{(1)}$ \\
\hline \multirow{4}{*}{$.1, .3, .7$} & 0 & -1.98 & .002 & -.002 & .021 & -.003 & .002 & 3.13 & .101 & .114 & .646 & .194 & .187 \\
\hline & .5 & 4.60 & .048 & .058 & .098 & .075 & -.064 & 10.27 & .521 & .511 & 1.00 & .846 & .724 \\
\hline & .7 & 7.14 & -.054 & 051 & .094 & .073 & .068 & 15.97 & .577 & .491 & .954 & .832 & .702 \\
\hline & .9 & 7.96 & .058 & .059 & .092 & -.071 & .056 & 17.98 & .618 & .635 & .994 & .782 & .697 \\
\hline \multirow{4}{*}{$.2, .6, .9$} & 0 & -.007 & .008 & -.006 & -.052 & -.010 & .008 & .272 & .197 & .142 & .523 & .298 & .355 \\
\hline & .5 & .070 & -.045 & .049 & .051 & -.056 & .042 & .787 & .311 & .530 & .610 & .688 & .546 \\
\hline & .7 & .077 & -.028 & -.032 & .041 & .038 & -.031 & .694 & .286 & .332 & .485 & .666 & .518 \\
\hline & .9 & -.080 & .039 & .035 & .041 & .037 & .031 & .731 & .240 & .421 & .515 & .507 & .577 \\
\hline \multirow{4}{*}{$.4, .7, .8$} & 0 & -.007 & .008 & -.006 & -.060 & -.009 & -.008 & .360 & .182 & .173 & .510 & .356 & .321 \\
\hline & .5 & -.074 & -.057 & .065 & .068 & -.062 & .058 & .953 & .585 & .691 & .818 & .712 & .745 \\
\hline & .7 & .059 & .043 & -.037 & -.046 & -.044 & .041 & .821 & .501 & .606 & .589 & .576 & .511 \\
\hline & .9 & .062 & .042 & .040 & -.040 & .041 & .037 & .725 & .496 & 519 & .554 & .529 & .495 \\
\hline \multirow{4}{*}{$0,1,1$} & 0 & -.004 & .002 & .002 & -.003 & .006 & .005 & .087 & .016 & .014 & .021 & .058 & .053 \\
\hline & .5 & -.009 & .005 & .002 & -.005 & -.009 & .008 & .082 & .041 & .046 & .071 & .091 & .078 \\
\hline & .7 & .005 & .007 & .009 & .006 & .006 & .006 & .072 & .048 & .034 & .038 & .095 & .083 \\
\hline & .9 & -.007 & .006 & .006 & -.006 & .005 & .005 & .081 & .033 & 049 & .026 & .053 & .062 \\
\hline
\end{tabular}


Table 3: Monte Carlo bias and SD of estimates of $\beta$ in model (2.1) with $q=1$ in cases (a)-(d) and (i)-(iv), with $n=128$, and 2000 replications

\begin{tabular}{|c|c|c|c|c|c|c|c||c|c|c|c|c|c|}
\hline & & \multicolumn{10}{|c||}{ Bias } & \multicolumn{6}{|c|}{$\mathrm{SD}$} \\
\hline$\gamma, \delta, \chi$ & $\rho$ & $\mathrm{OLS}$ & $\mathrm{NB}_{4}$ & $\mathrm{NB}_{5}$ & $\mathrm{IV}_{w}$ & $\mathrm{IV}_{(.5)}$ & $\mathrm{IV}_{(1)}$ & $\mathrm{OLS}$ & $\mathrm{NB}_{4}$ & $\mathrm{NB}_{5}$ & $\mathrm{IV}_{w}$ & $\mathrm{IV}_{(.5)}$ & $\mathrm{IV}_{(1)}$ \\
\hline & 0 & 1.26 & .001 & .002 & .014 & .002 & .002 & 2.17 & .092 & .085 & .575 & .139 & .128 \\
& .5 & 4.33 & .040 & .040 & .097 & .074 & .063 & 9.69 & .504 & .463 & .985 & .779 & .713 \\
$.1, .3, .7$ & .7 & 6.91 & -.051 & .048 & .092 & .071 & .069 & 15.95 & .509 & .463 & 938 & .725 & .633 \\
& .9 & 7.86 & .053 & .056 & .090 & .060 & .048 & 17.58 & .536 & .587 & .929 & 728 & .602 \\
\hline \multirow{5}{*}{$.2, .6, .9$} & 0 & .004 & -.005 & .005 & .040 & .008 & .008 & .118 & .112 & .201 & .412 & .301 & .265 \\
& .5 & .060 & .038 & .040 & .043 & .050 & .034 & .711 & .145 & .349 & .542 & .425 & .502 \\
& .7 & .062 & .025 & .039 & .034 & .040 & .029 & .618 & .267 & .344 & .339 & .626 & .433 \\
& .9 & .068 & .037 & .031 & .031 & .033 & .030 & .606 & .303 & .401 & .478 & 460 & .493 \\
\hline \multirow{5}{*}{$4, .7, .8$} & 0 & .003 & -.007 & .005 & .050 & .007 & .006 & .180 & .157 & .187 & .611 & .281 & .242 \\
& .5 & .045 & .054 & .061 & -.051 & .058 & -.051 & .675 & .622 & .674 & .601 & .690 & .653 \\
& .7 & .052 & .032 & .029 & .038 & .029 & .024 & .794 & .435 & .514 & .523 & .490 & .481 \\
& .9 & .059 & .040 & .035 & .034 & .038 & .036 & .638 & .481 & .492 & .521 & .505 & .421 \\
\hline & 0 & .001 & .002 & .002 & .001 & -.005 & .004 & .010 & .010 & .012 & .019 & .051 & .047 \\
& .5 & .003 & .002 & .001 & .004 & .005 & -.004 & .055 & .012 & .029 & .091 & .071 & .095 \\
& .7 & .001 & .006 & -.009 & .006 & .006 & -.004 & .045 & .029 & .020 & 026 & .076 & .067 \\
& .9 & .002 & .003 & .003 & -.005 & .005 & .004 & .052 & .029 & .044 & .021 & .059 & .051 \\
\hline
\end{tabular}


Table 4: Convergence rates of deviations of estimates from $\beta_{1}, \beta_{2}, \beta_{3}$ in model (2.1) with $q=3 . X=$ "inconsistent".

\begin{tabular}{|c|c|c|c|}
\hline Parameter & OLS & $\mathrm{IV}_{(.5)}$ & $\mathrm{IV}_{(1)}$ \\
\hline$\beta_{1}$ & $X$ & $n^{-.3} / \log n$ & $n^{-.3}$ \\
$\beta_{2}$ & $n^{-.4}$ & $n^{-.6} / \log n$ & $n^{-.6}$ \\
$\beta_{3}$ & $n^{-.7}$ & $n^{-.9} / \log n$ & $n^{-.9}$ \\
\hline
\end{tabular}

Table 5: Monte Carlo bias and SD of estimates of $\beta_{1}$ in model (2.1) with $q=3$ in cases (I)-(IV), with $n=64$ and 128, and 2000 replications

\begin{tabular}{|c|c|c|c|c||c|c|c|}
\hline & & \multicolumn{3}{|c||}{ Bias } & \multicolumn{3}{c|}{ SD } \\
\hline$n$ & $\rho_{3}$ & OLS & $\operatorname{IV}_{(.5)}$ & $\operatorname{IV}_{(1)}$ & OLS & IV $_{(.5)}$ & $\operatorname{IV}_{(1)}$ \\
\hline \multirow{5}{*}{64} & 0 & 4.93 & .021 & -.010 & 14.6 & .224 & .139 \\
& .5 & -5.88 & .085 & .070 & 19.7 & .869 & .748 \\
& .7 & 4.01 & .073 & .064 & 17.7 & .777 & .638 \\
& .9 & 5.27 & .076 & .065 & 18.8 & .764 & .665 \\
\hline \multirow{6}{*}{128} & 0 & 4.37 & -.014 & .009 & 7.36 & .108 & .101 \\
& .5 & 4.45 & -.080 & .067 & 8.69 & .817 & .720 \\
& .6 & -4.94 & -.069 & .062 & 16.5 & .700 & .635 \\
& .9 & 5.34 & .072 & .064 & 18.1 & .719 & .628 \\
\hline
\end{tabular}


Table 6: Monte Carlo bias and SD of estimates of $\beta_{2}$ in model (2.1) with $q=3$ in cases (I)-(IV), with $n=64$ and 128, and 2000 replications

\begin{tabular}{|c|c|c|c|c||c|c|c|}
\hline & & \multicolumn{3}{|c||}{ Bias } & \multicolumn{3}{c|}{ SD } \\
\hline$n$ & $\rho_{3}$ & OLS & $\mathrm{IV}_{(.5)}$ & $\mathrm{IV}_{(1)}$ & OLS & $\mathrm{IV}_{(.5)}$ & $\mathrm{IV}_{(1)}$ \\
\hline \multirow{6}{*}{64} & 0 & .016 & .016 & .009 & .298 & .217 & .110 \\
& .5 & -.093 & .078 & -.062 & 1.02 & .692 & .731 \\
& .7 & .089 & .064 & .054 & .996 & .731 & .616 \\
& .9 & .098 & .063 & .051 & 1.21 & .704 & .631 \\
\hline \multirow{6}{*}{128} & 0 & .015 & .011 & .009 & .253 & .103 & .089 \\
& .5 & .088 & .074 & .062 & .962 & .652 & .697 \\
& .6 & .087 & .060 & .047 & .901 & .627 & .568 \\
& .9 & .096 & .060 & -.046 & .928 & .616 & .593 \\
\hline
\end{tabular}

Table 7: Monte Carlo bias and SD of estimates of $\beta_{3}$ in model (2.1) with $q=3$ in cases (I)-(IV), with $n=64$ and 128 , and 2000 replications

\begin{tabular}{|c|c|c|c|c||c|c|c|}
\hline & & \multicolumn{3}{|c||}{ Bias } & \multicolumn{3}{c|}{ SD } \\
\hline$n$ & $\rho_{3}$ & OLS & $\mathrm{IV}_{(.5)}$ & $\mathrm{IV}_{(1)}$ & OLS & $\mathrm{IV}_{(.5)}$ & $\mathrm{IV}_{(1)}$ \\
\hline \multirow{6}{*}{64} & 0 & .009 & .014 & .008 & .199 & .183 & .108 \\
& .5 & .078 & -.057 & -.046 & .825 & .673 & .581 \\
& .7 & .082 & .056 & .045 & .889 & .693 & .652 \\
& .9 & .083 & .057 & .042 & .890 & .711 & .534 \\
\hline \multirow{6}{*}{128} & 0 & .008 & .009 & -.005 & .130 & .137 & .091 \\
& .5 & .072 & .053 & .042 & .797 & .648 & .548 \\
& .6 & .074 & -.051 & -.041 & .828 & .674 & .639 \\
& .9 & .079 & .054 & .040 & .838 & .733 & .522 \\
\hline
\end{tabular}


Table 8: Monte Carlo bias and SD of estimates of $\beta_{1}$ in model (2.1) with $q=8, n=64,128$, with $\rho_{i}=\rho_{i j} \equiv 0$, and 2000 replications

\begin{tabular}{|c|c|c|c|c||c|c|c|}
\hline & & \multicolumn{3}{|c||}{ Bias } & \multicolumn{3}{c|}{ SD } \\
\hline$\gamma, \delta$ & $n$ & OLS & $I V_{(.5)}$ & $I V_{(1)}$ & OLS & $I V_{(.5)}$ & $I V_{(1)}$ \\
\hline $.1, .4$ & 64 & -.955 & .011 & .010 & 1.13 & .156 & .149 \\
& 128 & .750 & .010 & .009 & .880 & .142 & .138 \\
\hline $.4, .7$ & 64 & .016 & .008 & -.008 & .155 & .131 & .133 \\
& 128 & .012 & .005 & .005 & .129 & .121 & .116 \\
\hline 0,1 & 64 & -.007 & .004 & .004 & .088 & .086 & .081 \\
& 128 & .003 & .003 & .002 & .042 & .036 & .038 \\
\hline
\end{tabular}

Table 9: Monte Carlo bias and SD of estimates of $\beta_{2}$ in model (2.1) with $q=8, n=64,128$, with $\rho_{i}=\rho_{i j} \equiv 0$, and 2000 replications

\begin{tabular}{|c|c|c|c|c||c|c|c|}
\hline & & \multicolumn{3}{|c||}{ Bias } & \multicolumn{3}{c|}{ SD } \\
\hline$\gamma, \delta$ & $n$ & OLS & $I V_{(.5)}$ & $I V_{(1)}$ & OLS & $I V_{(.5)}$ & $I V_{(1)}$ \\
\hline $.1, .4$ & 64 & .883 & -.011 & .011 & 1.13 & .181 & .168 \\
& 128 & .697 & .010 & -.010 & .805 & .134 & .122 \\
\hline $.4, .7$ & 64 & .019 & .006 & -.006 & .170 & .126 & .130 \\
& 128 & .011 & .003 & .002 & .148 & .118 & .115 \\
\hline 0,1 & 64 & -.009 & .004 & .003 & .093 & .073 & .072 \\
& 128 & .005 & -.002 & .002 & .046 & .038 & .031 \\
\hline
\end{tabular}


Table 10: Monte Carlo bias and SD of estimates of $\beta$ in model (2.1) with $q=1$, in cases (a')-(d'), with $n=64,128$, and 2000 replications

\begin{tabular}{|c|c|c|c|c|c|c|c||c|c|c|c|c|c|}
\hline & & \multicolumn{9}{|c||}{ Bias } & \multicolumn{6}{c|}{$\mathrm{SD}$} \\
\hline$\delta, \chi$ & $n$ & $\mathrm{OLS}$ & $\mathrm{NB}_{4}$ & $\mathrm{NB}_{5}$ & $\mathrm{IV}_{w}$ & $\mathrm{IV}_{(.5)}$ & $\mathrm{IV}_{(1)}$ & $\mathrm{OLS}$ & $\mathrm{NB}_{4}$ & $\mathrm{NB}_{5}$ & $\mathrm{IV}_{w}$ & $\mathrm{IV}_{(.5)}$ & $\mathrm{IV}_{(1)}$ \\
\hline $.3, .7$ & 64 & -.016 & .014 & .013 & -.014 & .014 & -.014 & .220 & .191 & .185 & .217 & .216 & .211 \\
& 128 & .013 & .011 & .011 & .011 & .010 & .010 & .171 & .163 & .168 & .170 & .164 & .159 \\
\hline $.6, .8$ & 64 & .047 & .038 & -.040 & .046 & .038 & .040 & .561 & .491 & .506 & .546 & .495 & .501 \\
& 128 & -.041 & .030 & .031 & -.040 & .033 & .031 & .519 & .427 & .437 & .521 & .504 & .501 \\
\hline $.7, .9$ & 64 & -.086 & -.072 & .076 & .083 & .079 & .074 & .683 & .574 & .580 & .670 & .649 & .624 \\
& 128 & .064 & .053 & -.052 & .058 & -.060 & .059 & .678 & .673 & .598 & .628 & .610 & .608 \\
\hline 1,1 & 64 & .098 & .080 & .080 & .084 & .082 & .081 & 1.01 & .808 & .855 & .970 & .932 & .878 \\
& 128 & -.065 & -.046 & .047 & .053 & .0 .50 & -.047 & .940 & .786 & .792 & .937 & .812 & .815 \\
\hline
\end{tabular}

Table 11: Monte Carlo bias and SD of estimates in model (2.1) with $q=1$ in cases (a)-(d), with $t_{5}$ errors, with $n=64,128$ and 2000 replications

\begin{tabular}{|c|c|c|c|c|c|c|c||c|c|c|c|c|c|}
\hline & & \multicolumn{10}{|c||}{ Bias } & \multicolumn{1}{c|}{ SD } \\
\hline$\gamma, \delta, \chi$ & $n$ & $\mathrm{OLS}$ & $\mathrm{NB}_{4}$ & $\mathrm{NB}_{5}$ & $\mathrm{IV}_{w}$ & $\mathrm{IV}_{(.5)}$ & $\mathrm{IV}_{(1)}$ & $\mathrm{OLS}$ & $\mathrm{NB}_{4}$ & $\mathrm{NB}_{5}$ & $\mathrm{IV}_{w}$ & $\mathrm{IV}_{(.5)}$ & $\mathrm{IV}_{(1)}$ \\
\hline $.1, .3, .7$ & 64 & 5.20 & .062 & .066 & .078 & .075 & .074 & 12.38 & 1.08 & 1.24 & 1.53 & 1.32 & 1.27 \\
& 128 & 4.90 & .048 & .047 & .099 & .079 & .044 & 10.85 & .963 & .912 & 1.38 & 1.12 & 1.01 \\
\hline $.2, .6, .9$ & 64 & .081 & .059 & .058 & .075 & .069 & .068 & 1.96 & .627 & .651 & .850 & .872 & .864 \\
& 128 & .078 & .042 & .044 & .047 & .052 & .054 & 1.53 & .539 & .494 & .800 & .761 & .797 \\
\hline $.4, .7, .8$ & 64 & .085 & .063 & .064 & .070 & .071 & .069 & 1.94 & 1.11 & 1.12 & 1.15 & 1.13 & 1.13 \\
& 128 & .049 & .054 & .064 & .052 & .060 & .053 & 1.34 & 1.02 & .985 & 1.18 & 1.08 & 1.09 \\
\hline $0,1,1$ & 64 & .007 & .006 & .005 & .008 & .007 & .006 & .212 & .191 & .207 & .234 & .219 & .211 \\
& 128 & .003 & .003 & .004 & .006 & .005 & .005 & .154 & .155 & .168 & .197 & .174 & .181 \\
\hline
\end{tabular}

